\title{
The influence of solar wind variability on magnetospheric ULF wave power
}

\author{
D. Pokhotelov ${ }^{1}$, I. J. Rae ${ }^{1}$, K. R. Murphy ${ }^{2}$, and I. R. Mann ${ }^{3}$ \\ ${ }^{1}$ Mullard Space Science Laboratory, UCL, Dorking, Surrey, UK \\ ${ }^{2}$ NASA Goddard Space Flight Center, Greenbelt, Maryland, USA \\ ${ }^{3}$ Department of Physics, University of Alberta, Edmonton, Alberta, Canada \\ Correspondence to: D. Pokhotelov (d.pokhotelov@ucl.ac.uk)
}

Received: 1 April 2015 - Revised: 7 May 2015 - Accepted: 8 May 2015 - Published: 8 June 2015

\begin{abstract}
Magnetospheric ultra-low frequency (ULF) oscillations in the Pc 4-5 frequency range play an important role in the dynamics of Earth's radiation belts, both by enhancing the radial diffusion through incoherent interactions and through the coherent drift-resonant interactions with trapped radiation belt electrons. The statistical distributions of magnetospheric ULF wave power are known to be strongly dependent on solar wind parameters such as solar wind speed and interplanetary magnetic field (IMF) orientation. Statistical characterisation of ULF wave power in the magnetosphere traditionally relies on average solar wind-IMF conditions over a specific time period. In this brief report, we perform an alternative characterisation of the solar wind influence on magnetospheric ULF wave activity through the characterisation of the solar wind driver by its variability using the standard deviation of solar wind parameters rather than a simple time average. We present a statistical study of nearly one solar cycle (1996-2004) of geosynchronous observations of magnetic ULF wave power and find that there is significant variation in ULF wave powers as a function of the dynamic properties of the solar wind. In particular, we find that the variability in IMF vector, rather than variabilities in other parameters (solar wind density, bulk velocity and ion temperature), plays the strongest role in controlling geosynchronous ULF power. We conclude that, although time-averaged bulk properties of the solar wind are a key factor in driving ULF powers in the magnetosphere, the solar wind variability can be an important contributor as well. This highlights the potential importance of including solar wind variability especially in studies of ULF wave dynamics in order to assess the efficiency of solar wind-magnetosphere coupling.
\end{abstract}

Keywords. Magnetospheric physics (solar windmagnetosphere interactions) - space plasma physics (waves and instabilities)

\section{Introduction}

Ultra-low frequency (ULF) waves in the Pc 4-5 range (45600 s period; 2-22 mHz; Jacobs et al., 1964) were theoretically described by Dungey and Southwood (1970) and originally characterised by ground-based magnetometer observations (Singer et al., 1977; Greenstadt et al., 1979). For instance, ULF oscillations can act to enhance radial diffusion serving as a diffusive mechanism for the energisation of radiation belt electrons and ions (Ozeke et al., 2012; Murphy et al., 2014). Also the resonant interaction between azimuthally drifting radiation belt electrons and standing Pc 4-5 ULF waves has been analysed both theoretically and numerically (Schulz and Lanzerotti, 1974; Elkington et al., 2003), and there is growing observational evidence that coherent drift resonance interactions between large-amplitude ULF waves and electrons contribute to electron acceleration in the radiation belts (Mann et al., 2013).

The interplanetary sources of standing ULF waves were proposed in the original works of Dungey and Southwood (1970), and ground magnetometer studies have confirmed the relations between solar wind parameters and the intensity of magnetospheric ULF waves. In particular, the solar wind speed has been shown to be the crucial parameter in controlling ULF power (Greenstadt et al., 1979; Engebretson et al., 1998; Mathie and Mann, 2001; Mann et al., 2004), especially in the dayside and morning sectors and peaking at 
magnetic shells $L=6-7$ (e.g. Rae et al., 2012). It has also been suggested that ULF pulsations in Pc 4-5 range could be directly driven by periodic solar wind pressure pulses (Kepko et al., 2002) though the physical mechanism of energy transfer across the bow shock and magnetopause remains unclear. The mechanisms of magnetopause energy transfer in the relevant frequency range (e.g. Sibeck, 1994) may involve solar wind pressure pulses, impulsive penetration, KelvinHelmholtz instability and flux transfer events, with each of those mechanisms requiring distinct solar wind driving properties. Some of those mechanisms may become dominant during specific phases of geomagnetic disturbances, during specific solar wind regime (i.e. slow or fast solar wind) and during specific phases of the solar cycle dominated by slow or fast solar wind flows (e.g. Kataoka and Miyoshi, 2006; Tsurutani et al., 2006).

In this study, we present a statistical characterisation of ULF wave power inside the magnetosphere parametrised by the variability of solar wind rather than by time-averaged solar wind properties. Since the magnetospheric ULF power is typically characterised by power spectral densities, i.e. by dynamic spectral characteristics, it seems reasonable to characterise the solar wind flow by its dynamic characteristics rather than by simple time averages. Earlier works outlined the importance of solar wind variability for the geoeffectiveness of solar wind flow (Snekvik et al., 2013) and for the intensification of relativistic electron fluxes in the outer radiation belts (Kataoka and Miyoshi, 2006; Hietala et al., 2014).

A formal statistical approach is taken to initially describe this solar wind variability by the standard deviations of solar wind parameters over a certain time interval. Quantiles of the standard deviations are then computed to estimate which of the solar wind variability parameters has a stronger impact on magnetospheric ULF power. The ULF power is characterised here by the power spectral density (PSD) in the Pc 4-5 frequency range measured at the geostationary orbit by magnetometer on-board the GOES spacecraft over a large portion of the solar cycle 23 (years 1996-2004). This approach allows conclusions to be made about the relative importance of different solar wind variabilities responsible for the intensification of ULF power in the magnetosphere. It is demonstrated that the ULF power in the magnetosphere is most sensitive to the variability of interplanetary magnetic field (IMF) (as characterised by the hourly standard deviation of IMF vector) and substantially less sensitive to the variability of other basic solar wind parameters (solar wind proton temperature, density and bulk velocity).

\section{Data sets}

\subsection{GOES ULF data}

We use data from the GOES East spacecraft, located at geostationary orbit around $357^{\circ}$ geomagnetic longitude and $11^{\circ}$ geomagnetic latitude. The data from GOES 8 (19962003) and GOES 12 (2003-2004), subsequently located at the GOES East slot, have been used. Following the approach of Rae et al. (2005), three-axis fluxgate magnetometer data at $1 \mathrm{~min}$ resolution have been rotated into field-aligned coordinates where the $z$ axis is aligned with the main magnetic field, with the $x$ axis pointing radially outwards from the Earth and $y$ axis pointing eastwards.

This coordinate system was constructed using a background running mean magnetic field estimate of $30 \mathrm{~min}$, and we use the same database used in this study, between 1996 to 2004, inclusive. Time intervals when the GOES spacecraft may have been located near, or outside of, the magnetopause have been excluded from this data set by estimating the magnetopause stand-off distance using simple pressure balance relation and excluding the hourly intervals when the magnetopause is expected to be within $8 R_{\mathrm{E}}$ radial distance. The PSDs were calculated using a $1 \mathrm{~h}$ fast Fourier transform, as in Rae et al. (2012) and Ozeke et al. (2012).

\subsection{Solar wind data}

We use data from the OMNI solar wind database http://omniweb.gsfc.nasa.gov, which provides solar wind and IMF parameters from the Advanced Composition Explorer (ACE) and Wind spacecraft, generated from 1 to 4 min resolution data time-shifted to upstream the Earth's bow shock (King and Papitashvili, 2005). Solar wind variability is characterised by the hourly standard deviations of IMF vector $\sigma\left(B_{\mathrm{V}}\right)$, solar wind ion temperature $\sigma\left(T_{\mathrm{SW}}\right)$, solar wind plasma density $\sigma\left(N_{\mathrm{SW}}\right)$, and solar wind bulk velocity $\sigma\left(V_{\mathrm{SW}}\right)$ provided by the OMNI database. The standard deviation of IMF vector is defined as the length of the vector formed from the standard deviations of IMF components, i.e. $\sigma\left(B_{\mathrm{V}}\right)=\left(\sigma\left(B_{x}\right)^{2}+\sigma\left(B_{y}\right)^{2}+\sigma\left(B_{z}\right)^{2}\right)^{1 / 2}$. The parameters are chosen to represent the basic properties of solar wind plasma without making any a priori assumptions about their relevance to specific solar wind-magnetosphere coupling mechanisms.

\section{Geosynchronous ULF wave power as a function of solar wind variability}

The data set of GOES PSDs has been split into four magnetic local time (MLT) sectors: midnight (21:00-03:00 MLT), dawn (03:00-09:00 MLT), noon (09:00-15:00 MLT) and dusk (15:00-21:00 MLT). From the data set of standard deviations of four solar wind parameters $-\sigma\left(B_{\mathrm{V}}\right), \sigma\left(T_{\mathrm{SW}}\right)$, $\sigma\left(N_{\mathrm{SW}}\right)$ and $\sigma\left(V_{\mathrm{SW}}\right)-$ the 6-quantiles (sextiles) of the distribution of solar wind parameters have been computed. The median values of GOES PSDs in each MLT sector are then computed in the intervals between each of the sextile for the four solar wind parameters. 

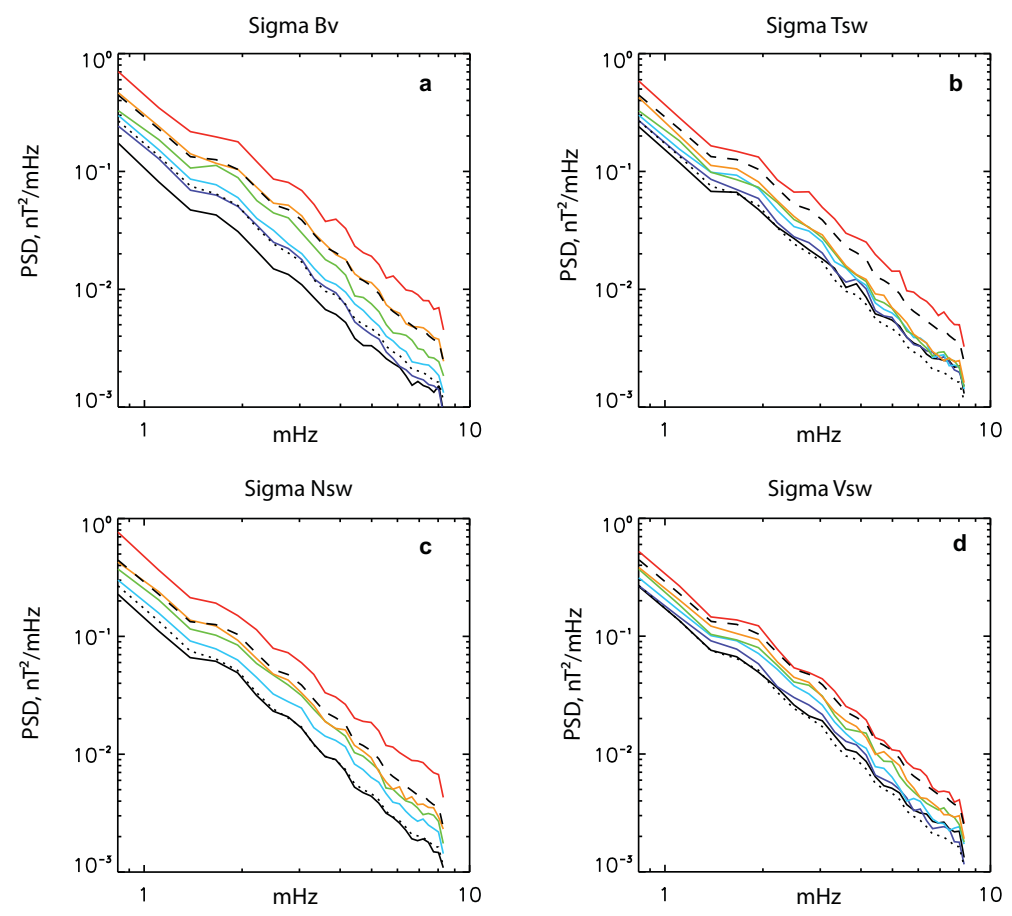

Figure 1. ULF wave power spectral density (PSD) as a function of solar wind variability. Median values of radial magnetic field ( $B_{x}$ component) PSDs are computed between sextiles of standard deviations of (a) interplanetary magnetic field vector, (b) solar wind ion temperature, (c) solar wind plasma density and (d) solar wind flow speed. The sextiles of solar wind variability are computed for the conditions when mean solar wind speed exceeds $450 \mathrm{~km} \mathrm{~s}^{-1}$. Dotted lines and dashed lines indicate the median values of GOES PSDs for all solar wind conditions when the mean solar wind speed exceeds 400 and $500 \mathrm{~km} \mathrm{~s}^{-1}$, respectively. For reference, the values of median and upper sextile of the solar wind parameters are given here: for $\sigma\left(B_{\mathrm{V}}\right)$ are 2.5 and $4.0 \mathrm{nT}$, for $\sigma\left(T_{\mathrm{SW}}\right)$ are $1.86 \times 10^{4}$ and $3.89 \times 10^{4} \mathrm{~K}$, for $\sigma\left(N_{\mathrm{SW}}\right)$ are 0.3 and $0.7 \mathrm{~cm}^{-3}$ and for $\sigma\left(V_{\mathrm{SW}}\right)$ are 9 and $16 \mathrm{~km} \mathrm{~s}^{-1}$, for the median and the upper sextiles, respectively.

Figure 1 presents the median values of GOES PSDs computed between sextiles of solar wind parameters, with colours in rainbow sequence (black to blue to red, solid lines) corresponding to higher sextiles. The sextiles of solar wind variability are computed for the conditions when mean solar wind speed exceeds $\left\langle V_{\mathrm{SW}}\right\rangle=450 \mathrm{~km} \mathrm{~s}^{-1}$. This threshold is chosen to separate the solar wind regimes dominated by fast and slow solar wind (Bothmer and Daglis, 2007). To illustrate the increase in ULF PSD power under higher mean solar wind speed known from previous studies (Pahud et al., 2009; Rae et al., 2012), the median values of GOES PSDs for all solar wind conditions whenever the mean solar wind speed exceeds 400 and $500 \mathrm{~km} \mathrm{~s}^{-1}$ are shown, respectively, by dotted lines and dashed lines. This demonstrates the increase in ULF PSD during geomagnetically disturbed periods.

The median spectral power presented in Fig. 1 is computed over the dayside MLT sector of the magnetosphere (MLT = 09:00-15:00). This MLT sector is chosen because earlier studies of the solar wind control (e.g. Rae et al., 2012) demonstrated the solar wind control to be most effective in the dayside and morning sectors. Other MLT sectors (analysed in this study but not shown here) demonstrate similar dependencies on solar wind variability. We only show the dependence of radial $\left(B_{x}\right)$ magnetic component corresponding to the poloidal Alfvén waves expected to be the most relevant to drift resonance interactions with radiation belt electrons (Ozeke and Mann, 2008). The other two magnetic components (azimuthal $B_{y}$ and compressional $B_{z}$ ) demonstrate similar solar wind dependencies supporting the conclusions below.

\section{Discussion and summary}

As summarised in Fig. 1, the higher solar wind variability corresponds to the higher values of ULF spectral power in the magnetosphere. It is also clear that the ULF power substantially increases during the periods of higher mean solar wind. This study is not aimed at quantifying the relative importance of mean solar wind parameters vs. their variabilities for the control of magnetospheric ULF power. However from the comparison of sextiles in Fig. 1 and the median PSD values above $\left\langle V_{\mathrm{SW}}\right\rangle=400$ and $500 \mathrm{~km} \mathrm{~s}^{-1}$, we can conclude that the solar wind variability has a pronounced effect on ULF power. It has to be pointed out that earlier studies (e.g. Pahud et al., 2009) used a substantially wider range of mean solar wind values (with the highest bin above $700 \mathrm{~km} \mathrm{~s}^{-1}$ ) thus showing higher values of ULF PSDs under extreme so- 
lar wind conditions compared to those in Fig. 1. The ULF PSDs shown in Fig. 1 do not demonstrate a clear peak at the fundamental harmonic of field line resonances that is consistent with earlier studies at the geostationary orbit. Junginger et al. (1984) presented mean electric and magnetic field PSDs calculated from 6 months of GEOS-2 data showing a broad peak in the electric field PSD around $3 \mathrm{mHz}$ (the fundamental harmonic of field line resonances) with no clear peak in the magnetic field PSD. Recent studies of the magnetic ULF PSDs using GOES data (e.g. Ozeke et al., 2012, 2014) also showed magnetic PSDs close to the power law, similar to those in Fig. 1. The small deviations from the power law around $1.5-2 \mathrm{mHz}$ seen in Fig. 1 are unlikely to be related to a field resonance frequency and could be either a feature of the magnetopause penetration or solar wind driving.

The data set presented here does not show particularly strong interdependencies, with the linear Pearson correlation coefficients between solar wind hourly means and standard deviations being in the range of $0.5-0.6$. However the interdependence between mean solar wind parameters and their variabilities can further complicate the analysis and needs to be addressed carefully.

The formal statistical approach used in this study allows one to assess the magnetospheric response to variability of different solar wind parameters without making an a priori assumptions about the physical mechanisms driving the magnetospheric ULF power. As seen from Figure 1, the response of ULF spectral power to the variability of different solar wind parameters differs dramatically, with the response to the variability in IMF vector being the most pronounced. Stronger ULF response to the variability of IMF $B_{\mathrm{V}}$ relative to other solar wind parameters suggests the importance of quasi-periodic IMF variations, such as those carried by high-speed solar wind streams (Tsurutani et al., 2006). This outlines the necessity of analysing the nature of variability of the solar wind under different solar wind conditions, such as fast and slow solar wind regime, as well as during different phases of interplanetary coronal mass ejections, such as sheath and ejecta, characterised by different levels of fluctuations (Hietala et al., 2014). A more comprehensive approach to the problem would be to characterise the solar wind variability by cross-correlations between different solar wind parameters thus allowing one to deduce the nature of solar wind variability, leading to the solar wind characteristics such as compressiveness or Alfvénicity (Tu and Marsch, 1995; Snekvik et al., 2013). Moreover this is important to analyse the impact of solar wind driving and variability on the azimuthal structure of excited ULF waves as the azimuthal wave number $(m)$ will strongly impact the resonance interactions with radiation belt particles (e.g. Ozeke et al., 2014) and the high- $m$ number waves may significantly contribute to the statistics (Anderson et al., 1990).

This study represents a first attempt to characterise the ULF power by variability of solar wind drivers rather than by average values of solar wind parameters over a certain inter- val. This demonstrates that the solar wind variability plays a noticeable role in the control of magnetospheric ULF power and this needs to be taken into account when considering the impact of different types of solar wind forcing, though the relative importance of solar wind mean parameters vs. their variabilities for the control of ULF power needs to addressed in a separate study.

Acknowledgements. D. Pokhotelov and I. J. Rae are supported by Science and Technology Facilities Council (STFC) grant ST/L000563/1; I. J. Rae is also supported by Natural Environment Research Council (NERC) grants NE/M00886X/1 and NE/L007495/1. K. R. Murphy is funded by a Canadian NSERC Postdoctoral Fellowship. We thank H. Singer and NOAA for the use of GOES magnetometer data, obtained from NASA CDAWeb (http://cdaweb.gsfc.nasa.gov/). Solar wind data were obtained from NASA OMNIWeb (http://omniweb.gsfc.nasa.gov/).

The topical editor L. Blomberg thanks K. Takahashi for help in evaluating this paper.

\section{References}

Anderson, B. J., Engebretson, M. J., Rounds, S. P., Zanetti, L. J., and Potemra, T. A.: A statistical study of Pc 3-5 pulsations observed by the AMPTE/CCE Magnetic Fields Experiment, 1. Occurrence distributions, J. Geophys. Res., 95, 10495-10523, doi:10.1029/JA095iA07p10495, 1990.

Bothmer, V. and Daglis, I. A.: Space Weather - Physics and Effects, Springer-Verlag, Berlin, 438 pp., 2007.

Dungey, J. W. and Southwood, D. J.: Ultra low frequency waves in the magnetosphere, Space Sci. Rev., 10, 672-688, doi:10.1007/BF00171551, 1970.

Greenstadt, E. W., Olson, J. V., Loewen, P. D., Singer, H. J., and Russell, C. T.: Correlation of $\mathrm{Pc} 3,4$, and 5 activity with solar wind speed, J. Geophys. Res., 84, 6694-6696, doi:10.1029/JA084iA11p06694,1979.

Elkington, S. R., Hudson, M. K., and Chan, A. A.: Resonant acceleration and diffusion of outer zone electrons in an asymmetric geomagnetic field, J. Geophys. Res., 108, 1116, doi:10.1029/2001JA009202, 2003.

Engebretson, M., Glassmeier, K., Stellmacher, M., Hughes, W., and Luhr, H.: The dependence of high-latitude Pc5 wave power on solar wind velocity and on the phase of highspeed solar wind streams, J. Geophys. Res., 103, 26271-26283, doi:10.1029/97JA03143, 1998.

Hietala, H., Kilpua, E. K. J., Turner, D. L., and Angelopoulos, V.: Depleting effects of ICME-driven sheath regions on the outer electron radiation belt, Geophys. Res. Lett., 41, 2258-2265, doi:10.1002/2014GL059551, 2014.

Jacobs, J. A., Kato, Y., Matsushita, S., and Troitskaya, V. A.: Classification of geomagnetic micropulsations, J. Geophys. Res., 69, 180-181, doi:10.1029/JZ069i001p00180, 1964.

Junginger, H., Geiger, G., Haerendel, G., Melzner, F., Amata, E., and Higel, B.: A statistical study of dayside magnetospheric electric field fluctuations with periods between 150 and $600 \mathrm{~s}, \mathrm{~J}$. Geophys. Res., 89, 5495-5505, doi:10.1029/JA089iA07p05495, 1984. 
Kataoka, R. and Miyoshi, Y.: Flux enhancement of radiation belt electrons during geomagnetic storms driven by coronal mass ejections and corotating interaction regions, Space Weather, 4, S09004, doi:10.1029/2005SW000211, 2006.

Kepko, L., Spence, H. E., and Singer, H. J.: ULF waves in the solar wind as direct drivers of magnetospheric pulsations, Geophys. Res. Lett., 29, 1197, doi:10.1029/2001GL014405, 2002.

King, J. H. and Papitashvili, N. E.: Solar wind spatial scales in and comparisons of hourly Wind and ACE plasma and magnetic field data, J. Geophys. Res., 110, A02104, doi: 10.1029/2004JA010649, 2005.

Mann, I. R., O'Brien, T. P., and Milling, D. K.: Correlations between ULF wave power, solar wind speed, and relativistic electron flux in the magnetosphere: solar cycle dependence, J. Atmos. Sol.-Terr. Phys., 66, 187-198, doi:10.1016/j.jastp.2003.10.002, 2004.

Mann, I. R., Lee, E. A., Claudepierre, S. G., Fennell, J. F., Degeling, A., Rae, I. J, Baker, D. N., Reeves, G. D., Spence, H. E., Ozeke, L. G., Rankin, R., Milling, D. K., Kale, A., Friedel, R. H. W., and Honary, F.: Discovery of the action of a geophysical synchrotron in the Earth's Van Allen radiation belts, Nature Comm., 4, 2795, doi:10.1038/ncomms3795, 2013.

Mathie, R. A. and Mann, I. R.: On the solar wind control of Pc5 ULF pulsation power at mid-latitudes: Implications for $\mathrm{MeV}$ electron acceleration in the outer radiation belt, J. Geophys. Res., 106, 29783-29796, doi:10.1029/2001JA000002, 2001.

Murphy, K. R., Mann, I. R., and Ozeke, L. G.: A ULF wave driver of ring current energization, Geophys. Res. Lett., 41, 6595-6602, doi:10.1002/2014GL061253, 2014.

Ozeke, L. G. and Mann, I. R.: Energization of radiation belt electrons by ring current ion driven ULF waves, J. Geophys. Res., 113, A02201, doi:10.1029/2007JA012468, 2008.

Ozeke, L. G., Mann, I. R., Murphy, K. R., Rae, I. J., Milling, D. K., Elkington, S. R., Chan, A. A., and Singer, H. J.: ULF wave derived radiation belt radial diffusion coefficients, J. Geophys. Res., 117, A04222, doi:10.1029/2011JA017463, 2012.

Ozeke, L. G., Mann, I. R., Murphy, K. R., Rae, I. J., and Milling, D. K.: Analytic expressions for ULF wave radiation belt radial diffusion coefficients, J. Geophys. Res., 119, 1587-1605, doi:10.1002/2013JA019204, 2014.
Pahud, D. M., Rae, I. J., Mann, I. R., Murphy, K. R., and Amalraj, V.: Ground-based Pc5 ULF wave power: Solar wind speed and MLT dependence, J. Atmos. Sol.-Terr. Phys., 71, 1082-1092, doi:10.1016/j.jastp.2008.12.004, 2009.

Rae, I. J., Donovan, E. F., Mann, I. R., Fenrich, F. R., Watt, C. E. J., Milling, D. K., Lester, M., Lavraud, B., Wild, J. A., Singer, H. J., Rème, H., and Balogh, A.: Evolution and characteristics of global Pc5 ULF waves during a high solar wind speed interval, J. Geophys. Res., 110, A12211, doi:10.1029/2005JA011007, 2005.

Rae, I. J., Mann, I. R., Murphy, K. R., Ozeke, L. G., Milling, D. K., Chan, A. A., Elkington, S. R., and Honary, F.: Ground-based magnetometer determination of in situ Pc4-5 ULF electric field wave spectra as a function of solar wind speed, J. Geophys. Res.,117, A04221, doi:10.1029/2011JA017335, 2012.

Schulz, M. and Lanzerotti, L. J.: Physics and Chemistry in Space. 7: Particle Diffusion in the Radiation Belts, Springer-Verlag, Berlin, 215 pp., 1974.

Sibeck, D. G.: Transient and quasi-periodic (5-15 Min) events in the outer magnetosphere, Am. Geophys. Union Geophys. Monogr. Ser., 81, 173-182, doi:10.1029/GM081p0173, 1994.

Singer, H. J., Russell, C. T., Kivelson, M. G., Greenstadt E. W., and Olson, J. V.: Evidence for the control of Pc 3,4 magnetic pulsations by the solar wind velocity, Geophys. Res. Lett., 4, 377-379, doi:10.1029/GL004i009p00377, 1977.

Snekvik, K., Tanskanen, E. I., and Kilpua, E. K. J.: An automated identification method for Alfvénic streams and their geoeffectiveness, J. Geophys. Res., 118, 5986-5998, doi:10.1002/jgra.50588, 2013.

Tsurutani, B. T., Gonzalez, W. D.. Gonzalez, A. L. C., Guarnieri, F. L., Gopalswamy, N., Grande, M., Kamide, Y., Kasahara, Y., Lu, G., Mann, I., McPherron, R., Soraas, F., and Vasyliunas, V.: Corotating solar wind streams and recurrent geomagnetic activity: A review, J. Geophys. Res., 111, A07S01, doi:10.1029/2005JA011273, 2006.

Tu, C. Y. and Marsch, E.: MHD structures, waves and turbulence in the solar wind: Observations and theories, Space Sci. Rev., 73, 1-210, doi:10.1007/BF00748891, 1995. 